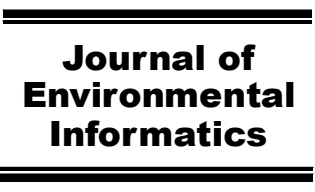

www.iseis.org/jei

\title{
An Extended Two-stage Stochastic Programming Approach for Water Resources Management under Uncertainty
}

\author{
J. Nematian* \\ Department of Industrial Engineering, Faculty of Mechanical Engineering, University of Tabriz, P.O. Box 51666-14766, Tabriz, Iran
}

Received June 29, 2014; revised April 4, 2015; accepted June 27, 2015; published online June 14, 2016

\begin{abstract}
In this study, extended two-stage stochastic programming with fuzzy variables is developed for water resources management under uncertainty. First, the problem is formulated and solved by an extended interval-parameter two-stage stochastic programming (ITSP) approach for retrieving water shortages. To this end, some alternatives are considered to retrieve the difference between the quantities of promised water-allocation targets and the actual allocated water. An extended ITSP is then developed for the problem under the fuzzy uncertainty by using fuzzy variables and solved using fuzzy chance-constrained programming based on the idea of possibility theory. Furthermore, an illustrative example is also given to clarify the methods discussed in this paper.
\end{abstract}

Keywords: water resources management, water shortages, extended two-stage programming, fuzzy chance-constrained programming, possibility theory

\section{Introduction}

In recent decades, problems including the efficient allocation of water supplies have been of increasing interest for water resource managers. In water resources management, the conflict-laden issues of water allocation among competing municipal, industrial and agricultural interests have intensified (Huang and Chang, 2003; Wang et al., 2003).

Furthermore, the growing population shifts cause a quick development in agriculture systems which leads to the shortage of water resources. In other words, increasing demand for water resources, shrinking water supplies, development of industries and agricultural interests specify the requirement of an efficient method for allocating water to multiple users.

Numerous mathematical optimization techniques (Li et al., 2007; Guo et al., 2010; Wang et al., 2011; Wang and Huang, 2011) have been developed to analyse the water resource allocation systems. For instance, Wang et al. (2011) proposed an interval-valued fuzzy linear programming with infinite $\alpha$-cuts method for environmental management under uncertainty. $\mathrm{Hu}$ et al. (2012) proposed an inexact fuzzy two-stage programming approach for supporting multi-water resources management under multi-uncertainties. Another inexact two-stage stochastic partial programming method is proposed by Fan et al.

* Corresponding author. Tel.: +98 4133392457 ; fax: +98 4133354153.

E-mail address: jnematian@tabrizu.ac.ir (J. Nematian).

ISSN: $1726-2135$ print/1684-8799 online

(C) 2016 ISEIS All rights reserved. doi: 10.3808/jei.201600334
(2012) for tackling uncertainties presented in form of intervals and partial probability distributions as an application to water resources management under uncertainty. Recently, Wang and Huang (2015) have presented a multi-level Taguchi-factorial two-stage stochastic programming approach for supporting water resources management under parameter uncertainties and their interactions.

Two-stage stochastic programming (TSP) is an effective technique for the analysis of problems in which an examination of policy scenarios is desired and the system data is characterrized by uncertainty. In TSP, an initial decision must be made before values of random variables are known, and then a corrective action can be taken after random variables have taken place.

TSP has been widely studied over the past decades (Wang and Adams, 1986; Birge and Louveaux, 1988; Eiger and Shamir, 1991; Ruszczynski and Swietanowski, 1997; Ferrero et al., 1998; Huang and Loucks, 2000; Maqsood et al., 2005; Guo et al., 2008; Wang and Huang, 2011). It has also been successfully applied to water resource management problems. Maqsood et al. (2005) offered an interval-parameter fuzzy two-stage stochastic program for water resources management. Guo et al. (2010) proposed a two-stage programming approach for water resources management under randomness and fuzziness. Furthermore, an interactive two-stage stochastic fuzzy programming (ITSFP) for water resources management was presented by Wang and Huang (2011).

Interval mathematical programming deals with uncertainties expressed as intervals with definite lower and upper bounds. Interval-parameter two-stage stochastic programming (ITSP) 
was presented due to insufficiencies of TSP (Huang and Loucks, 2000; Maqsood et al. 2005; Li and Huang, 2008). For instance, Huang (1996) employed an interval-parameter model for water quality management and provided its application to a case study of water pollution control planning within an agricultural system. For water resources management under uncertainty, $\mathrm{Li}$ et al. (2006) presented an interval-parameter multi-stage stochastic programming model, and more recently, Li and Huang (2008) proposed an interval-parameter two-stage stochastic nonlinear programming.

ITSP could not only tackle uncertainties expressed as random variables and intervals but also investigate a variety of policy scenarios. However, this method has remarkable limitations in handling possibilistic uncertainty or indicating the risk of violating the constraints of the problem.

In most real-world problems, the parameters may often be given as subjective information expressed as fuzzy sets, and several new methods have been developed to solve the problems with fuzzy parameters. Jairaj and Vedula (2000) optimized a multi-reservoir system using fuzzy programming and Maqsood et al. (2005) proposed a model derived by incorporating the concepts of interval-parameter and fuzzy programming techniques within a two-stage stochastic optimization framework. Jiménez et al. (2007) proposed an interactive fuzzy resolution method for solving linear programming problems with fuzzy parameters and Nie et al. (2007) introduced a hybrid interval-parameter fuzzy robust programming approach for waste management planning under uncertainty.

For water resources management, Wang and Huang (2011) proposed ITSFP through incorporating an interactive fuzzy resolution method within an inexact two-stage stochastic programming framework. ITSFP could handle dual uncertainties expressed as fuzzy boundary intervals that exist in the objective function and the left- and right-hand sides of constraint and analyse a variety of policy scenarios with different levels of economic penalties when the promised policy targets are violated. A new class of fuzzy stochastic optimization models presented by Wang and Watada (2011) is two-stage fuzzy stochastic programming with Value-at-Risk criteria.

In this paper, new extended methods are presented to solve the problems of water shortages. These methods include some alternatives such as other reservoirs to retrieve water shortages to reach the water allocation target. The main aim of this paper is to introduce new approaches for the problem, in a way that optimizes the system net benefit and gives optimal solutions that also choose proper alternatives for retrieving water shortages.

This paper presents two extended approaches. First, an extended ITSP approach is presented to water resources management for retrieving water shortages. Then, the extended ITSP is developed considering fuzzy parameters for the problem under fuzziness. The advantage of these extended approaches over the conventional type is considering some alternatives for retrieving water shortages when the water demands are not completely satisfied by seasonal flows which cause irreparable damages especially to industries, and users have to either ob- tain water from higher-priced resources or curtail their development plans.

The remainder of this paper is organized as follows. Section 2 proposes an extended ITSP approach for retrieving water shortages. In Section 3, an extended ITSP model with fuzzy variables is presented, and new methods are proposed to solve it. In Section 4, our computational results are reported and an illustrative example is also solved and analysed to clarify the described methods. Finally, Section 5 contains conclusions and discussions of future research.

\section{An Extension of ITSP}

In this section, a basic study is discussed to pave the way for presenting an extended approach for water resources programming under uncertainty. Indeed, we introduce a new extended approach under uncertain framework to retrieve the water shortages of users.

The problem of water resources management concerns with effective allocation of water resources among competing multiple users: an agricultural sector, a municipality and an industrial unit, and the main objective is to maximize the system benefit. In such problem, a prescribed quantity of water is promised to each user according to the average amount of water usage in past years and the water flow levels are uncertain, expressed as random variables with a known probabilistic distribution.

This problem consists of two stages. In the first stage, a decision must be undertaken before the values of random variables are defined, and then in the second stage, a corrective action can be taken after the disclosure of random variables and this leads to a TSP approach.

Thus, the real amount of the allocated water differs from the optimized water-allocation target promised to each user and the water demands may not thoroughly be satisfied. The shortage amount will be the optimized water-allocation target minus the actual allocation amount.

To solve the problem through the linear programming method, the distribution of total seasonal flow is approximated by discrete values $q_{j}$ with probabilities $p_{j}(j=1, \ldots, n)$. Therefore, this problem is formulated as a TSP model as follows:

Maximize

$f=\sum_{i=1}^{m} N B_{i} W_{i}-\sum_{i=1}^{m} \sum_{j=1}^{n} p_{j} C_{i} S_{i j}$

subject to

$$
\begin{aligned}
& \sum_{i=1}^{m}\left(W_{i}-S_{i j}\right)(1+\delta) \leq q_{j}, \quad \forall j \\
& S_{i j} \leq W_{i} \leq W_{i \max }, \quad \forall i, j \\
& S_{i j} \geq 0, \quad \forall i, j
\end{aligned}
$$


where $f=$ system benefit $(\$) ; N B_{i}=$ net benefit to user $i$ per $\mathrm{m}^{3}$ of water allocated $\left(\$ / \mathrm{m}^{3}\right) ; W_{i}=$ water-allocation target that is promised to user $i\left(\mathrm{~m}^{3}\right)$ (first-stage decision variables); $C_{i}=$ shortage cost to user $i$ per $\mathrm{m}^{3}$ of water shortage $\left(\$ / \mathrm{m}^{3}\right) ; S_{i j}=$ shortage of water to user $i$ when the seasonal flow is $q_{j}\left(\mathrm{~m}^{3}\right)$ (second-stage decision variables); $\delta$ = rate of water loss during transportation; $q_{j}=$ seasonal flow with probability $p_{j}$; $W_{i \max }=$ maximum allowable allocation amount for user $i\left(\mathrm{~m}^{3}\right)$; $n=$ total number of flow levels; $m=$ total number of water users; $i=$ water user, $i=1,2,3$,where $i=1$ for the municipality, $i=2$ for the industrial user, and $i=3$ for the agricultural sector.

The seasonal water flows that will be available for allocation are uncertain, so it seems difficult to suppose a definite amount for shortages, costs, benefits and other parameters. In addition, a manager is not able to promise a certain amount of water allocation to each user and in most cases, definition of the parameters with a probabilistic distribution seems impossible since they did not obey a known distribution. In the ITSP approach, parameters/variables are expressed as intervals defined by upper and lower bounds. Consider the following formulation of the problem as an ITSP model:

\section{Maximize}

$f^{ \pm}=\sum_{i=1}^{m} N B_{i}^{ \pm} W_{i}^{ \pm}-\sum_{i=1}^{m} \sum_{j=1}^{n} p_{j} C_{i}^{ \pm} S_{i j}^{ \pm}$

subject to

$\sum_{i=1}^{m}\left(W_{i}^{ \pm}-S_{i j}^{ \pm}\right)\left(1+\delta^{ \pm}\right) \leq q_{j}^{ \pm}, \quad \forall j$

$S_{i j}^{ \pm} \leq W_{i}^{ \pm} \leq W_{i \max }^{ \pm}, \quad \forall i, j$

$S_{i j}^{ \pm} \geq 0, \quad \forall i, j$

where $N B_{i}^{ \pm}, W_{i}^{ \pm}, C_{i}^{ \pm}, S_{i j}^{ \pm}, \delta^{ \pm}$and $W_{i \max }^{ \pm}$are interval parameters/variables. A number with a known upper and lower bound but with unknown distribution information is defined as an interval (Maqsood et al., 2005). For example, $S_{i j}^{ \pm}$is an interval decision variable and $S_{i j}^{-}$and $S_{i j}^{+}$are upper and lower bounds of $S_{i j}^{ \pm}$, respectively, and $S_{i j}^{ \pm}=\left[S_{i j}^{-}, S_{i j}^{+}\right]$.

Now, the conventional ITSP is developed and a corrective method is presented to satisfy water shortages that users and managers face with them. As said before, water shortages occur when the seasonal water flows do not suffice the promised water-allocation target to each user, which means that promised amount for water allocation exceeds the available water. In such cases, users will have to utilize supplementary resources to satisfy their water needs. In the following problem, an extended ITSP method is proposed to retrieve water shortages:

\section{Problem 1}

Maximize

$$
\begin{aligned}
f^{ \pm}= & \sum_{i=1}^{m} N B_{i}^{ \pm} W_{i}^{ \pm}-\sum_{i=1}^{m} \sum_{j=1}^{n} p_{j} C_{i}^{ \pm} S_{i j}^{ \pm} \\
& -\sum_{i=1}^{m} \sum_{j=1}^{n} p_{j} \sum_{k=1}^{l} E_{i k}^{ \pm} \Delta T_{i k}^{ \pm} x_{i j k}
\end{aligned}
$$

subject to

Water availability constraints:

$\sum_{i=1}^{m}\left(W_{i}^{ \pm}-S_{i j}^{ \pm}\right)\left(1+\delta^{ \pm}\right) \leq q_{j}^{ \pm}, \quad \forall j$

Allowable water allocation constraints:

$S_{i j}^{ \pm} \leq W_{i}^{ \pm} \leq W_{i \max }^{ \pm}, \quad \forall i, j$

Constraints that prevent users to exceed capacity of alternatives:

$\sum_{j=1}^{n} x_{i j k} \leq 1, \quad \forall i, k$

Constraints for retrieving water shortages:

$\sum_{k=1}^{l} \Delta T_{i k}^{ \pm} x_{i j k} \geq S_{i j}^{ \pm}, \quad \forall i, j$

Non-negativity constraints:

$S_{i j}^{ \pm} \geq 0, \quad \forall i, j$

Binary constraints for using alternatives:

$x_{i j k} \in\{0,1\}, \quad \forall i, j, k$

where $E_{i k}^{ \pm}=$cost of increasing $1 \mathrm{~m}^{3}$ water for user $i$ while using alternative $k\left(\$ / \mathrm{m}^{3}\right) ; l=$ total number of alternatives; $\Delta T_{i k}^{ \pm}=$ available amount of water for user $i$ by using alternative $k\left(\mathrm{~m}^{3}\right)$; $x_{i j k}$ is a binary decision variable that takes value 1 if user $i$ uses alternative $k$ when the seasonal flow is $j$.

Furthermore, the other parameters and variables of this problem are similar to those of the problem formulating based on the ITSP approach.

\subsection{Solution Method}

According to Huang (1996), problem 1 is divided into two deterministic mixed integer programming (MIP) sub-models. In the first sub-model, the goal is to reach the upper bound of the objective-function value $\left(f^{+}\right)$, so the parameters and variables are set in their lower or upper bounds in a way that the upper bound for the objective function occurs. Conversely, the second sub-model is based on the lower bound of the objectivefunction value $\left(f^{-}\right)$. According to Huang and Loucks (2000), $W_{i}^{ \pm}$is converted to a deterministic value derived from $W_{i}^{ \pm}=$ $W_{i}^{-}+\Delta W_{i} z_{i}$, where $\Delta W_{i}=W_{i}^{+}-W_{i}^{-}, z_{i} \in[0,1]$ and $z_{i}$ are decision variables that are used to determine $W_{i}^{ \pm}$. 
The first sub-model corresponding to $f^{+}$can be formulated as follows:

Problem 2

Maximize

$$
\begin{aligned}
f^{+}= & \sum_{i=1}^{m} N B_{i}^{+}\left(W_{i}^{-}+\Delta W_{i} z_{i}\right)-\sum_{i=1}^{m} \sum_{j=1}^{n} p_{j} C_{i}^{-} S_{i j}^{-} \\
& -\sum_{i=1}^{m} \sum_{j=1}^{n} p_{j} \sum_{k=1}^{l} E_{i k}^{-} \Delta T_{i k}^{-} x_{i j k}
\end{aligned}
$$

subject to

$$
\begin{aligned}
& \sum_{i=1}^{m}\left(W_{i}^{-}+\Delta W_{i} z_{i}-S_{i j}^{-}\right) \leq q_{j}^{+}, \quad \forall j \\
& S_{i j}^{-} \leq W_{i}^{-}+\Delta W_{i} z_{i} \leq W_{i \max }^{+}, \quad \forall i, j \\
& \sum_{j=1}^{n} x_{i j k} \leq 1, \quad \forall i, k \\
& \sum_{k=1}^{l} \Delta T_{i k}^{-} x_{i j k} \geq S_{i j}^{-}, \quad \forall i, j \\
& S_{i j}^{-} \geq 0, \quad \forall i, j \\
& x_{i j k} \in\{0,1\}, \quad \forall i, j, k \\
& 0 \leq z_{i} \leq 1, \quad \forall i
\end{aligned}
$$

where $S_{i j}^{-}, z_{i}$ and $x_{i j k}$ are decision variables. Let $S_{i j o p t}^{-}, z_{i o p t}$ and $x_{i j k \text { opt }\left(f^{+}\right)}$are the optimal solutions of the first sub-model. Then, the optimized water-allocation targets can be obtained by calculating $W_{\text {iopt }}^{ \pm}=W_{i}^{-}+\Delta W_{i} z_{\text {iopt }}$.

According to Huang (1996), the sub-model corresponding to $f^{-}$can be formulated as follows in which the calculated amounts of $S_{i j o p t}^{-}, z_{i o p t}$ and $x_{i j k \text { opt }\left(f^{+}\right)}$are used:

Problem 3

Maximize

$$
\begin{aligned}
f^{-}= & \sum_{i=1}^{m} N B_{i}^{-}\left(W_{i}^{-}+\Delta W_{i} z_{i o p t}\right)-\sum_{i=1}^{m} \sum_{j=1}^{n} p_{j} C_{i}^{+} S_{i j}^{+} \\
& -\sum_{i=1}^{m} \sum_{j=1}^{n} p_{j} \sum_{k=1}^{l} E_{i k}^{+} \Delta T_{i k}^{+} x_{i j k}
\end{aligned}
$$

subject to

$$
\begin{aligned}
& \sum_{i=1}^{m}\left(W_{i}^{-}+\Delta W_{i} z_{i o p t}-S_{i j}^{+}\right) \leq q_{j}^{-}, \forall j \\
& S_{i j}^{+} \leq W_{i}^{-}+\Delta W_{i} z_{i o p t} \leq W_{i \max }^{-}, \quad \forall i, j \\
& \sum_{j=1}^{n} x_{i j k} \leq 1, \quad \forall i, k
\end{aligned}
$$

$$
\sum_{k=1}^{l} \Delta T_{i k}^{+} x_{i j k} \geq S_{i j}^{+}, \quad \forall i, j
$$

$S_{i j}^{+} \geq 0, \quad \forall i, j$

$x_{i j k} \geq x_{i j k \text { opt }\left(f^{+}\right)}, \quad \forall i, j, k$

$S_{i j}^{+} \geq S_{i j o p t}^{-}, \quad \forall i, j$

$x_{i j k} \in\{0,1\}, \quad \forall i, j, k$

where $S_{i j}^{+}$and $x_{i j k}$ are decision variables. Problems 2 and 3 are deterministic MIP problems and the optimal solutions of problem 1 are:

$S_{i j o p t}^{ \pm}=\left[S_{i j o p t}^{-}, S_{i j o p t}^{+}\right], \quad \forall i, j$

$f_{\text {opt }}^{ \pm}=\left[f_{\text {opt }}^{-}, f_{\text {opt }}^{+}\right]$

where $S_{\text {ijopt }}^{-}, f_{\text {opt }}^{+}$are the optimal solution of problem 2, and $S_{i j \text { opt }}^{+}, f_{\text {opt }}^{-}$are those of problem 3 . Therefore, the actual allocated water scheme supplied by seasonal flows may be calculated as follows:

$A_{i j o p t}^{ \pm}=W_{i o p t}^{ \pm}-S_{i j o p t}^{ \pm}, \forall i, j$

To handle the uncertainty of parameters of the water resource management problem which has imprecise and vague properties and interval variables cannot explain this uncertainty, an extended fuzzy approach will be introduced based on possibility theory.

\section{An Extended ITSP with Fuzzy Variables}

Fuzzy optimization is a flexible method, which can deal with the real-world problems in the existence of indistinct information. For the problem under fuzzy uncertainty, an extended ITSP is developed by fuzzy variables (called fuzzy extended ITSP) and solved by applying fuzzy chance-constrained programming (FCCP) based on the idea of possibility theory.

In this section, a new TSP model is introduced in which the parameters are fuzzy numbers and the decision variables will be obtained as intervals. The rate of loss and the flow levels with probability $p_{j}$ are fuzzy numbers. Furthermore, the differrence between the promised water-allocation targets and the actual allocated water is retrieved by some alternatives.

By an explanation of Puri and Ralescu (1986), LR fuzzy number $\tilde{A}$ is defined by the following membership function:

$$
\tilde{A}(X)=\left\{\begin{array}{lcc}
L\left(\frac{A^{0}-x}{A^{-}}\right) & \text {if } & A^{0}-A^{-} \leq x<A^{0} \\
1 & \text { if } & A^{0} \leq x \leq A^{1} \\
R\left(\frac{x-A^{1}}{A^{+}}\right) & \text {if } & A^{1}<x \leq A^{1}+A^{+}
\end{array}\right.
$$


where $\left[A^{0}, A^{1}\right]$ denotes the peak of fuzzy number and $A^{-}, A^{+}$ indicate the left and right spread respectively; $L, R:[0,1] \rightarrow$ $[0,1]$ with $L(0)=R(0)=1$ and $L(1)=R(1)=0$ are strictly decreasing, continuous functions. The LR fuzzy number may also be represented as $\tilde{A}=\left(A^{0}, A^{1}, A^{-}, A^{+}\right)_{L R}$.

A fuzzy extended ITSP model for the water resources management problem is presented by the following problem:

Problem 4

Maximize

$$
\begin{aligned}
& \tilde{f}^{ \pm}=\sum_{i=1}^{m} N \tilde{B}_{i} W_{i}^{ \pm}-\sum_{i=1}^{m} \sum_{j=1}^{n} p_{j} \tilde{C}_{i} S_{i j}^{ \pm} \\
& -\sum_{i=1}^{m} \sum_{j=1}^{n} p_{j} \sum_{k=1}^{l} \tilde{E}_{i k} \Delta T_{i k}^{ \pm} x_{i j k}
\end{aligned}
$$

subject to

$$
\begin{aligned}
& \sum_{i=1}^{m}\left(W_{i}^{ \pm}-S_{i j}^{ \pm}\right)(1+\tilde{\delta}) \leq \tilde{q}_{j}, \quad \forall j \\
& S_{i j}^{ \pm} \leq W_{i}^{ \pm} \leq W_{i \max }^{ \pm}, \quad \forall i, j \\
& \sum_{j=1}^{n} x_{i j k} \leq 1, \quad \forall i, k \\
& \sum_{k=1}^{l} \Delta T_{i k}^{ \pm} x_{i j k} \geq S_{i j}^{ \pm}, \quad \forall i, j \\
& x_{i j k} \in\{0,1\}, \quad \forall i, j, k \\
& S_{i j}^{ \pm} \geq 0, \quad \forall i, j
\end{aligned}
$$

where $\tilde{\delta}=\left(\delta^{0}, \delta^{1}, \beta, \gamma\right)_{L R}, N \tilde{B}_{i}=\left(N B_{i}^{0}, N B_{i}^{1}, \alpha_{i}, \lambda_{i}\right)_{L R}, \quad \tilde{C}_{i}=($ $\left.C_{i}^{0}, C_{i}^{1}, \rho_{i}, \varphi_{i}\right)_{L R}, \tilde{q}_{j}=\left(q_{j}^{0}, q_{j}^{0}, \beta_{j}, \gamma_{j}\right)_{L R}$ and $\tilde{E}_{i k}=\left(E_{i k}^{0}, E_{i k}^{1}, \alpha_{i k}\right.$, $\left.\beta_{i k}\right)_{L R}$ are LR fuzzy numbers.

\subsection{Fuzzy Chance-constrained Programming}

According to Huang and Loucks (2000), let $W_{i}^{ \pm}=W_{i}^{-}$ $+\Delta W_{i} z_{i}$, where $\Delta W_{i}=W_{i}^{+}-W_{i}^{-}$and $z_{i} \in[0,1]$, and $z_{i}$ are decision variables that are used for identifying an optimized set of target values $\left(W_{i}^{ \pm}\right)$. Based on the method presented by Huang (1996), problem 4 may be divided into two sub-models. The first sub-model corresponding to the upper bound of the objecttive function $\left(\tilde{f}^{+}\right)$is formulated as follows:

Problem 5

Maximize

$$
\begin{aligned}
\tilde{f}^{+}= & \sum_{i=1}^{m} N \tilde{B}_{i}\left(W_{i}^{-}+\Delta W_{i} z_{i}\right)-\sum_{i=1}^{m} \sum_{j=1}^{n} p_{j} \tilde{C}_{i} S_{i j}^{-} \\
& -\sum_{i=1}^{m} \sum_{j=1}^{n} p_{j} \sum_{k=1}^{l} \tilde{E}_{i k} \Delta T_{i k}^{-} x_{i j k}
\end{aligned}
$$

subject to

$\sum_{i=1}^{m}\left(W_{i}^{-}+\Delta W_{i} z_{i}-S_{i j}^{-}\right)(1+\tilde{\delta}) \leq \tilde{q}_{j}, \quad \forall j$

$$
\begin{aligned}
& S_{i j}^{-} \leq W_{i}^{-}+\Delta W_{i} z_{i} \leq W_{i \max }^{+}, \quad \forall i, j \\
& \sum_{j=1}^{n} x_{i j k} \leq 1, \quad \forall i, k \\
& \sum_{k=1}^{l} \Delta T_{i k}^{-} x_{i j k} \geq S_{i j}^{-}, \quad \forall i, j \\
& x_{i j k} \in\{0,1\}, \quad \forall i, j, k \\
& S_{i j}^{-} \geq 0, \quad \forall i, j \\
& 0 \leq z_{i} \leq 1, \quad \forall i
\end{aligned}
$$

The objective function and the first constraint of problem 5 contain fuzzy parameters and hence have fuzzy properties. The rest of constraints are identical to constraints of the extended ITSP problem. The way for transforming this problem, which is a fuzzy programming model, to a deterministic MIP model is using possibility measures to the objective function and the constraints.

As the problem 5 is not well defined, a lower bound will be considered to the objective function and a degree of possibility is defined to the constraints whose coefficients are fuzzy variables. Then, we use a FCCP method based on the possibility theory to maximize the lower bound of the objective function subject to the constraints.

From extension principle of Zadeh, $\tilde{f}^{+} \geq f^{+}$is a fuzzy event defined on the possibility space $(\Theta, p(\Theta), P o s)$, whose possibility is:

$$
\operatorname{Pos}\left(\tilde{f}^{+} \geq f^{+}\right)=\sup _{y_{1}, y_{2} \in \mathfrak{R}}\left\{\min \left\{\mu_{\tilde{f}^{+}}\left(y_{1}\right), \mu_{f^{+}}\left(y_{2}\right)\right\} \mid y_{1} \geq y_{2}\right\}
$$

where Pos represents possibility.

Furthermore, $\sum_{i=1}^{m}\left(W_{i}^{-}+\Delta W_{i} z_{i}-S_{i j}^{-}\right)(1+\tilde{\delta}) \leq \tilde{q}_{j}$ is a fuzzy event defined on $(\Theta, p(\Theta), P o s)$, whose possibility is:

$$
\begin{aligned}
& \operatorname{Pos}\left(\sum_{i=1}^{m}\left(W_{i}^{-}+\Delta W_{i} z_{i}-S_{i j}^{-}\right)(1+\tilde{\delta}) \leq \tilde{q}_{j}\right) \\
& =\sup _{y_{1}, y_{2} \in \Re}\left\{\min \left\{\mu_{\tilde{a}_{j}^{-}}\left(y_{1}\right), \mu_{\tilde{q}_{j}}\left(y_{2}\right)\right\} \mid y_{1} \leq y_{2}\right\}
\end{aligned}
$$

where $\tilde{a}_{j}^{-}=\sum_{i=1}^{m}\left(W_{i}^{-}+\Delta W_{i} z_{i}-S_{i j}^{-}\right)(1+\tilde{\delta})$.

By using the idea of FCCP based on possibility measures, a possibility-based model of problem 5 can be defined as follows:

Problem 6

Maximize $f^{+}$

subject to

$$
\operatorname{Pos}\left(\tilde{f}^{+} \geq f^{+}\right) \geq \eta
$$




$$
\begin{aligned}
& \operatorname{Pos}\left(\sum_{i=1}^{m}\left(W_{i}^{-}+\Delta W_{i} z_{i}-S_{i j}^{-}\right)(1+\tilde{\delta}) \leq \tilde{q}_{j}\right) \geq \eta, \quad \forall j \\
& S_{i j}^{-} \leq W_{i}^{-}+\Delta W_{i} z_{i} \leq W_{i \max }^{+}, \quad \forall i, j \\
& \sum_{j=1}^{n} x_{i j k} \leq 1, \quad \forall i, k \\
& \sum_{k=1}^{l} \Delta T_{i k}^{-} x_{i j k} \geq S_{i j}^{-}, \quad \forall i, j \\
& x_{i j k} \in\{0,1\}, \quad \forall i, j, k \\
& S_{i j}^{-} \geq 0, \quad \forall i, j \\
& 0 \leq z_{i} \leq 1, \quad \forall i
\end{aligned}
$$

where the lower bound $f^{+}$is maximized and the possibility measures in Equations (55) and (56) are used to fuzzy events of problem 5. Furthermore, $\eta$ is a permissible possibility level.

In what follows, we try to transform Equations (55) and (56) into deterministic constraints and obtain the following theorem:

Theorem 1:

For any decision vector, it holds that:

$$
\begin{aligned}
& \text { I) } \quad \operatorname{Pos}\left(\tilde{f}^{+} \geq f^{+}\right) \geq \eta \\
& \Leftrightarrow \sum_{i=1}^{m}\left(N B_{i}^{1}+R^{*}(\eta) \lambda_{i}\right)\left(W_{i}^{-}+\Delta W_{i} z_{i}\right) \\
& +\sum_{i=1}^{m} \sum_{j=1}^{n} p_{j}\left(-C_{i}^{0}+R^{*}(\eta) \rho_{i}\right) S_{i j}^{-} \\
& \quad+\sum_{i=1}^{m} \sum_{j=1}^{n} p_{j} \sum_{k=1}^{l}\left(-E_{i k}^{0}+R^{*}(\eta) \alpha_{i k}\right) \Delta T_{i k}^{-} x_{i j k} \geq f^{+} \\
& \text {II) } \quad \operatorname{Pos}\left(\sum_{i=1}^{m}\left(W_{i}^{-}+\Delta W_{i} z_{i}-S_{i j}^{-}\right)(1+\tilde{\delta}) \leq \tilde{q}_{j}\right) \geq h \\
& \Leftrightarrow \sum_{i=1}^{m}\left(W_{i}^{-}+\Delta W_{i} z_{i}-S_{i j}^{-}\right)\left(1+\delta^{0}-L^{*}(h) \beta\right) \leq q_{j}^{1}+R^{*}(h) \gamma_{j}
\end{aligned}
$$

where $L^{*}$ and $R^{*}$ are pseudo inverse functions defined as $L^{*}(\lambda)$ $=\sup \{t \mid L(t) \geq \lambda\}, R^{*}(\lambda)=\sup \{t \mid R(t) \geq \lambda\}$. The proof of this theorem is given in Appendix.

As a direct result of theorem 1, problem 6 is equivalently written as follows:

Problem 7

$$
\begin{aligned}
\text { Maximize } & \sum_{i=1}^{m}\left(N B_{i}^{1}+R^{*}(\eta) \lambda_{i}\right)\left(W_{i}^{-}+\Delta W_{i} z_{i}\right) \\
& +\sum_{i=1}^{m} \sum_{j=1}^{n} p_{j}\left(-C_{i}^{0}+R^{*}(\eta) \rho_{i}\right) S_{i j}^{-} \\
& +\sum_{i=1}^{m} \sum_{j=1}^{n} p_{j} \sum_{k=1}^{l}\left(-E_{i k}^{0}+R^{*}(\eta) \alpha_{i k}\right) \Delta T_{i k}^{-} x_{i j k}
\end{aligned}
$$

subject to

$$
\begin{aligned}
& \sum_{i=1}^{m}\left(W_{i}^{-}+\Delta W_{i} z_{i}-S_{i j}^{-}\right)\left(1+\delta^{0}-L^{*}(\eta) \beta\right) \leq q_{j}^{1}+R^{*}(\eta) \gamma_{j}, \forall j \\
& S_{i j}^{-} \leq W_{i}^{-}+\Delta W_{i} z_{i} \leq W_{i \max }^{+}, \quad \forall i, j \\
& \sum_{j=1}^{n} x_{i j k} \leq 1, \quad \forall i, k \\
& \sum_{k=1}^{l} \Delta T_{i k}^{-} x_{i j k} \geq S_{i j}^{-}, \quad \forall i, j \\
& x_{i j k} \in\{0,1\}, \quad \forall i, j, k \\
& S_{i j}^{-} \geq 0, \quad \forall i, j \\
& 0 \leq z_{i} \leq 1, \quad \forall i
\end{aligned}
$$

In the above problem, $S_{i j}^{-}, z_{i}$ and $x_{i j k}$ are decision variables and the optimal water-allocation targets are determined by $W_{i}^{ \pm}=W_{i}^{-}+\Delta W_{i} z_{i}$. The optimal solutions of problem 7, shown by $S_{i j o p t}^{-}, z_{i o p t}, x_{i j k \text { opt }}$ are used to write the second sub-model of problem 4.

According to Huang (1996), the second sub-model corresponding to the lower bound of the objective function value $\left(\tilde{f}^{-}\right)$ is formulated by the following problem:

\section{Problem 8}

Maximize

$$
\begin{aligned}
\tilde{f}^{-}= & \sum_{i=1}^{m} N \tilde{B}_{i}\left(W_{i}^{-}+\Delta W_{i} z_{i o p t}\right)-\sum_{i=1}^{m} \sum_{j=1}^{n} p_{j} \tilde{C}_{i} S_{i j}^{+} \\
& -\sum_{i=1}^{m} \sum_{j=1}^{n} p_{j} \sum_{k=1}^{l} \tilde{E}_{i k} \Delta T_{i k}^{+} x_{i j k}
\end{aligned}
$$

subject to

$$
\sum_{i=1}^{m}\left(W_{i}^{-}+\Delta W_{i} z_{\text {iopt }}-S_{i j}^{+}\right)(1+\tilde{\delta}) \leq \tilde{q}_{j}, \quad \forall j
$$

$S_{i j}^{+} \leq W_{i}^{-}+\Delta W_{i} z_{\text {iopt }} \leq W_{i \max }^{-}, \quad \forall i, j$

$\sum_{j=1}^{n} x_{i j k} \leq 1, \quad \forall i, k$

$\sum_{k=1}^{l} \Delta T_{i k}^{+} x_{i j k} \geq S_{i j}^{+}, \quad \forall i, j$

$x_{i j k} \geq x_{i j k o p t\left(f^{+}\right)}, \quad \forall i, j, k$

$S_{i j}^{+} \geq S_{i j o p t}^{-}, \quad \forall i, j$

$x_{i j k} \in\{0,1\}, \quad \forall i, j, k$ 
where $S_{i j}^{+}$and $x_{i j k}$ are decision variables. In problem $8, \tilde{f}^{-} \geq f^{-}$ and $\sum_{i=1}^{m^{m}}\left(W_{i}^{-}+\Delta W_{i} z_{i o p t}-S_{i j}^{+}\right)(1+\tilde{\delta}) \leq \tilde{q}_{j}$ are fuzzy events defined on possibility spaced, whose possibility are defined as:

$$
\begin{gathered}
\operatorname{Pos}\left(\tilde{f}^{-} \geq f^{-}\right)=\sup _{y_{1}, y_{2} \in \Re}\left\{\min \left\{\mu_{\tilde{f}^{-}}\left(y_{1}\right), \mu_{f^{-}}\left(y_{2}\right)\right\} \mid y_{1} \geq y_{2}\right\} \\
\operatorname{Pos}\left(\sum_{i=1}^{m}\left(W_{i}^{-}+\Delta W_{i} z_{i o p t}-S_{i j}^{+}\right)(1+\tilde{\delta}) \leq \tilde{q}_{j}\right) \\
=\sup _{y_{1}, y_{2} \in \Re}\left\{\min \left\{\mu_{\tilde{a}_{j}^{+}}\left(y_{1}\right), \mu_{\tilde{q}_{j}}\left(y_{2}\right)\right\} \mid y_{1} \leq y_{2}\right\}
\end{gathered}
$$

where $\tilde{a}_{j}^{+}=\sum_{i=1}^{m}\left(W_{i}^{-}+\Delta W_{i} z_{i o p t}-S_{i j}^{+}\right)(1+\tilde{\delta})$.

Like the first sub-model, by using the idea of FCCP based on possibility measures, a possibility-based model of problem 8 is defined as follows:

Problem 9

Maximize $f^{-}$

subject to

$$
\begin{aligned}
& \operatorname{Pos}\left(\tilde{f}^{-} \geq f^{-}\right) \geq \eta \\
& \operatorname{Pos}\left(\sum_{i=1}^{m}\left(W_{i}^{-}+\Delta W_{i} z_{i o p t}-S_{i j}^{+}\right)(1+\tilde{\delta}) \leq \tilde{q}_{j}\right) \geq \eta, \quad \forall j \\
& S_{i j}^{+} \leq W_{i}^{-}+\Delta W_{i} z_{i o p t} \leq W_{i \max }^{-}, \quad \forall i, j \\
& \sum_{j=1}^{n} x_{i j k} \leq 1, \quad \forall i, k \\
& \sum_{k=1}^{l} \Delta T_{i k}^{+} x_{i j k} \geq S_{i j}^{+}, \quad \forall i, j \\
& x_{i j k} \geq x_{i j k \text { opt }\left(f^{+}\right)}, \quad \forall i, j, k \\
& S_{i j}^{+} \geq S_{i j o p t}^{-}, \quad \forall i, j \\
& x_{i j k} \in\{0,1\}, \quad \forall i, j, k
\end{aligned}
$$

In order to transform Equations (84) and (85) into linear constraints, we obtain the following corollary:

Corollary 1:

For any decision vector, it holds that:

$$
\begin{aligned}
& \text { I) } \quad \operatorname{Pos}\left(\tilde{f}^{-} \geq f^{-}\right) \geq \eta \\
& \Leftrightarrow \sum_{i=1}^{m}\left(N B_{i}^{1}+R^{*}(\eta) \lambda_{i}\right)\left(W_{i}^{-}+\Delta W_{i} z_{\text {iopt }}\right) \\
& +\sum_{i=1}^{m} \sum_{j=1}^{n} p_{j}\left(-C_{i}^{0}+R^{*}(\eta) \rho_{i}\right) S_{i j}^{+} \\
& +\sum_{i=1}^{m} \sum_{j=1}^{n} p_{j} \sum_{k=1}^{l}\left(-E_{i k}^{0}+R^{*}(\eta) \alpha_{i k}\right) \Delta T_{i k}^{+} x_{i j k} \geq f^{-}
\end{aligned}
$$

$$
\begin{aligned}
& \text { II) } \operatorname{Pos}\left(\sum_{i=1}^{m}\left(W_{i}^{-}+\Delta W_{i} z_{i o p t}-S_{i j}^{+}\right)(1+\tilde{\delta}) \leq \tilde{q}_{j}\right) \geq h \\
& \Leftrightarrow \sum_{i=1}^{m}\left(W_{i}^{-}+\Delta W_{i} z_{i o p t}-S_{i j}^{+}\right)\left(1+\delta^{0}-L^{*}(h) \beta\right) \\
& \leq q_{j}^{1}+R^{*}(h) \gamma_{j}
\end{aligned}
$$

Consequently, from corollary 1, problem 9 is equivalently transformed to the following problem:

Problem 10

$$
\begin{aligned}
\text { Maximize } & \sum_{i=1}^{m}\left(N B_{i}^{1}+R^{*}(\eta) \lambda_{i}\right)\left(W_{i}^{-}+\Delta W_{i} z_{i o p t}\right) \\
& +\sum_{i=1}^{m} \sum_{j=1}^{n} p_{j}\left(-C_{i}^{0}+R^{*}(\eta) \rho_{i}\right) S_{i j}^{+} \\
& +\sum_{i=1}^{m} \sum_{j=1}^{n} p_{j} \sum_{k=1}^{l}\left(-E_{i k}^{0}+R^{*}(\eta) \alpha_{i k}\right) \Delta T_{i k}^{+} x_{i j k}
\end{aligned}
$$

subject to

$$
\begin{aligned}
& \sum_{i=1}^{m}\left(W_{i}^{-}+\Delta W_{i} z_{i o p t}-S_{i j}^{+}\right)\left(1+\delta^{0}-L^{*}(\eta) \beta\right) \leq q_{j}^{1}+R^{*}(\eta) \gamma_{j} \\
& S_{i j}^{+} \leq W_{i}^{-}+\Delta W_{i} z_{i o p t} \leq W_{i \max }^{-}, \quad \forall i, j \\
& \sum_{j=1}^{n} x_{i j k} \leq 1, \quad \forall i, k \\
& \sum_{k=1}^{l} \Delta T_{i k}^{+} x_{i j k} \geq S_{i j}^{+}, \quad \forall i, j \\
& x_{i j k} \geq x_{i j k \text { opt }\left(f^{+}\right)}, \quad \forall i, j, k \\
& S_{i j}^{+} \geq S_{i j o p t}^{-}, \quad \forall i, j \\
& x_{i j k} \in\{0,1\}, \quad \forall i, j, k
\end{aligned}
$$

Problems 7 and 10 are deterministic MIP problems which can be solved by one of the MIP solvers. The optimal solutions of problem 4 are:

$S_{i j o p t}^{ \pm}=\left[S_{i j o p t}^{-}, S_{i j o p t}^{+}\right], \quad \forall i, j$

$f_{\text {opt }}^{ \pm}=\left[f_{\text {opt }}^{-}, f_{\text {opt }}^{+}\right]$

where $S_{i j o p t}^{-}$and $f_{\text {opt }}^{+}$are the solutions of problem 7 , and $S_{i j o p t}^{+}$and $f_{\text {opt }}^{-}$are those of problem 10. As said before, the actual allocated water scheme may be calculated as:

$A_{i j o p t}^{ \pm}=W_{i o p t}^{ \pm}-S_{i j o p t}^{ \pm}, \forall i, j$

In our extended approaches, the shortage amount, which is the difference between the promised water allocation target 
and the actual allocated water, can be also retrieved by some supplementary reservoirs distinguished by $x_{i j k \text { opt }}$.

In the following steps, we summarize an algorithm for solving the problem discussed in this section:

Algorithm 1

Data Entry:

Step 0. Define fuzzy parameters of problem 4 by using information of experts or decision makers.

Model structure:

Step 1. According to Huang (1996):

Convert problem 4 to sub-problems 5 and 8 .

Step 2. Apply the FCCP method based on possibility measures:

Convert sub-problems 5 and 8 to problems 6 and 9, respectively.

Step 3. Obtain theorem 1and corollary 1.

Convert problem 6 to problem 7 (use theorem 1).

Convert Problem 9 to problem 10 (use corollary 1).

Solution Procedure:

Step 4. Solve the obtained MIP problems by one of the MIP solvers. Then, the optimal solution of problem 4 is obtained by $S_{i j o p t}^{ \pm}=\left[S_{i j o p t}^{-}, S_{i j o p t}^{+}\right], A_{i j o p t}^{ \pm}=W_{i o p t}^{ \pm}-S_{i j o p t}^{ \pm}$and $W_{i o p t}^{ \pm}=W_{i}^{-}+\Delta W_{i} z_{\text {iopt }}$.

\section{Illustrative Example}

\subsection{Problem Definition}

To emphasize the implementation of this study, an illustrative example of the water resources management in Tabriz city, an urban area in the north-western of Iran, has been considered. All data has been collected through a survey of experts or decision makers of water resources management. In this problem, the water resources manager is responsible for allocating water resources between three users: a municipality, an industrial unit, and an agricultural sector (as shown in Figure 1) in an area of Tabriz city. It is assumed that seasonal flows, which vary within low to high, are uncertain with known probabilities. The boundary intervals for the seasonal flows under different probability levels are presented in Table 1 . Moreover, Table 1 shows the maximum allowable water allocation, the waterallocation target, the net benefit to user $i$ per $\mathrm{m}^{3}$ of water allo- cated and the shortage cost to user $i$ per $\mathrm{m}^{3}$ of water not delivered.

If the seasonal flows are insufficient and the whole allocation target for promised water is not delivered, users will have to use water stores of three neighbour areas. By using these alternatives, the cost of increasing $1 \mathrm{~m}^{3}$ of water for user $i$ and available amount of water for user $i$ are given in Table 2 . In Figure 1, “ $\longrightarrow$ " indicates water allocated by alternatives and " $\Longrightarrow$ " indicates actual allocated water $A_{i j}^{ \pm}, i=1,2,3$ suplied by seasonal flows.

The problem is how to effectively allocate the water supply to multiple users to maximize system net benefit and obtain optimal solutions for choosing the most proper alternative.

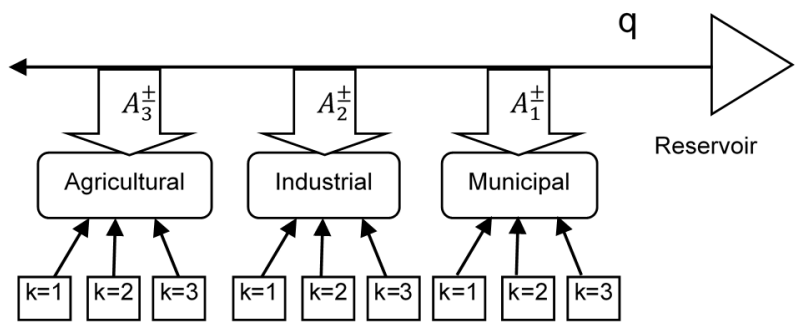

Figure 1. Diagram of water-allocation to multiple users.

Table 1. Related Economic Data $\left(\$ / \mathrm{m}^{3}\right)$ and Seasonal Flows $\left(10^{3} \mathrm{~m}^{3}\right)$ under Different Probability

\begin{tabular}{|c|c|c|c|}
\hline & \multicolumn{3}{|c|}{ Users } \\
\hline & $\begin{array}{l}\text { Municipal } \\
(\mathrm{i}=1)\end{array}$ & $\begin{array}{l}\text { Industrial } \\
(\mathrm{i}=2)\end{array}$ & $\begin{array}{l}\text { Agricultural } \\
(\mathrm{i}=3)\end{array}$ \\
\hline $\begin{array}{l}\text { Net benefit for water } \\
\text { allocation }\left(N B^{ \pm}\right)\end{array}$ & {$[85,105]$} & {$[40,50]$} & {$[23,30]$} \\
\hline $\begin{array}{l}\text { Water-allocation target } \\
\left(W_{i}^{ \pm}\right)\end{array}$ & {$[1,2.5]$} & {$[2,4]$} & {$[3.5,6]$} \\
\hline $\begin{array}{l}\text { Maximum allowable } \\
\text { water allocation } \\
\left(W_{i \max }^{ \pm}\right)\end{array}$ & 8 & 8 & 8 \\
\hline \multirow[t]{3}{*}{ Shortage cost $\left(C_{i}^{ \pm}\right)$} & {$[20,32]$} & {$[21,26]$} & {$[23,40]$} \\
\hline & \multicolumn{3}{|c|}{ Flow level } \\
\hline & $\begin{array}{l}\text { Low } \\
(\mathrm{j}=1)\end{array}$ & $\begin{array}{l}\text { Medium } \\
(\mathrm{j}=2)\end{array}$ & $\begin{array}{l}\text { High } \\
(\mathrm{j}=3)\end{array}$ \\
\hline Seasonal flow $\left(q_{j}^{ \pm}\right)$ & {$[3.2,4.2]$} & {$[7,11]$} & {$[14,18]$} \\
\hline Probability & 0.2 & 0.6 & 0.2 \\
\hline
\end{tabular}

Table 3. Fuzzy Parameters of Economic Data $\left(\$ / \mathrm{m}^{3}\right)$ and Seasonal Flows $\left(10^{3} \mathrm{~m}^{3}\right)$.

\begin{tabular}{|c|c|c|c|c|}
\hline & & \multicolumn{3}{|c|}{ Users } \\
\hline & & Municipal $(i=1)$ & Industrial $(\mathrm{i}=2)$ & Agricultural $(\mathrm{i}=3)$ \\
\hline$N \tilde{B}_{i}=\left(N B_{i}^{0}, N B_{i}^{1}, \alpha_{i}, \lambda_{i}\right)_{L R}$ & & $(85,105,10,15)_{L R}$ & $(40,50,5,6)_{L R}$ & $(23,30,4,7)_{L R}$ \\
\hline \multirow[t]{3}{*}{$\tilde{C}_{i}=\left(C_{i}^{0}, C_{i}^{1}, \rho_{i}, \varphi_{i}\right)_{L R}$} & & $(20,32,1.5,1.25)_{L R}$ & $(21,26,0.3,0.2)_{L R}$ & $(23,40,0.8,1)_{L R}$ \\
\hline & & Flow level & & \\
\hline & & Low $(\mathrm{j}=1)$ & Medium $(j=2)$ & High $(j=3)$ \\
\hline \multirow[t]{3}{*}{$\tilde{q}_{j}=\left(q_{j}^{0}, q_{j}^{1}, \beta_{j}, \gamma_{j}\right)_{L R}$} & & $(3.2,4.2,0.5,1)_{L R}$ & $(7,11,0.5,1)_{L R}$ & $(14,18,0.25,1)_{L R}$ \\
\hline & & User & & \\
\hline & Alternative & Municipal $(\mathrm{i}=1)$ & Industrial $(\mathrm{i}=2)$ & Agricultural (i = 3) \\
\hline \multirow{3}{*}{$\tilde{E}_{i k}=\left(E_{i k}^{0}, E_{i k}^{1}, \alpha_{i k}, \beta_{i k}\right)_{L R}$} & $\mathrm{k}=1$ & $(12,20,0.3,0.4)_{L R}$ & $(10,25,0.12,0.3)_{L R}$ & $(8,25,0.2,0.6)_{L R}$ \\
\hline & $\mathrm{k}=2$ & $(20,40,0.7,0.8)_{L R}$ & $(25,38,0.25,0.5)_{L R}$ & $(20,45,0.35,0.8)_{L R}$ \\
\hline & $\mathrm{k}=3$ & $(10,20,0.2,0.1)_{L R}$ & $(10,18,0.7,0.25)_{L R}$ & $(5,15,0.25,0.3)_{L R}$ \\
\hline
\end{tabular}


Table 2. Cost of Increasing $1 \mathrm{~m}^{3}$ of Water $\left(\$ / \mathrm{m}^{3}\right)$ and Available Amount of Water $\left(10^{3} \mathrm{~m}^{3}\right)$ for Users by Using Alternatives

\begin{tabular}{lllll}
\hline & & \multicolumn{3}{c}{ Users } \\
\cline { 2 - 5 } & Alternative & $\begin{array}{l}\text { Municipal } \\
(\mathrm{i}=1)\end{array}$ & $\begin{array}{l}\text { Industrial } \\
(\mathrm{i}=2)\end{array}$ & $\begin{array}{l}\text { Agricultural } \\
(\mathrm{i}=3)\end{array}$ \\
\hline \multirow{4}{*}{$E_{i k}^{ \pm}$} & $\mathrm{k}=1$ & {$[12,20]$} & {$[10,25]$} & {$[8,25]$} \\
& $\mathrm{k}=2$ & {$[20,40]$} & {$[25,38]$} & {$[20,45]$} \\
& $\mathrm{k}=3$ & {$[10,20]$} & {$[10,18]$} & {$[5,15]$} \\
\hline \multirow{4}{*}{$\Delta T_{i k}^{ \pm}$} & & \multicolumn{3}{c}{ User } \\
\cline { 2 - 5 } & $\mathrm{k}=2$ & {$[1,3]$} & {$[1,3.5]$} & {$[3,4.2]$} \\
& $\mathrm{k}=1$ & {$[\mathrm{i}=1)$} & $\begin{array}{l}\text { Andustrial } \\
(\mathrm{i}=2)\end{array}$ & Agricultural \\
& $\mathrm{k}=3$ & {$[2,5]$} & {$[3,4.5]$} & {$[0.5,1.5]$} \\
\hline
\end{tabular}

Consider the same case, but suppose that the parameters have a high level of uncertainty which is not proper to define them as interval variables. In this situation, parameters of this problem have uncertain properties determined by fuzzy variables and all of the elements of uncertain parameters are given in Table 3. Suppose that $R(x)=L(x)=1-x, \tilde{\delta}=(0.07,0.15$, $0.01,0.03)_{L R}$ and $\eta=0.7$.

\subsection{Result Analysis}

Without considering alternatives to retrieve users' water shortage, apply the general ITSP method to the described problem and solve the obtained sub-problems by LINGO 8.0. The optimized water-allocation targets, water shortages, and waterallocation scheme are collected in Table 4.

Table 4. Optimal Solutions of the General ITSP Method under Optimized Water-allocation Targets $\left(10^{3} \mathrm{~m}^{3}\right)$

\begin{tabular}{|c|c|c|c|c|}
\hline & $\begin{array}{l}\text { Proba- } \\
\text { bility }\end{array}$ & $\begin{array}{l}\text { Muni- } \\
\text { cipal } \\
(i=1)\end{array}$ & $\begin{array}{l}\text { Indus- } \\
\text { trial } \\
(\mathrm{i}=2)\end{array}$ & $\begin{array}{l}\text { Agri- } \\
\text { cultural } \\
(\mathrm{i}=3)\end{array}$ \\
\hline$W_{\text {iopt }}^{ \pm}$ & & 2.5 & 4 & 6 \\
\hline \multicolumn{5}{|c|}{$S_{i j ~ o p t}^{ \pm}$under 3 flow levels: } \\
\hline $\begin{array}{l}\text { Low } \\
(j=1)\end{array}$ & 0.2 & 2.5 & 4 & {$[1.8,2.8]$} \\
\hline $\begin{array}{l}\text { Medium } \\
(\mathrm{j}=2)\end{array}$ & 0.6 & 1.5 & {$[0,4]$} & 0 \\
\hline $\begin{array}{l}\text { High } \\
(\mathrm{j}=3)\end{array}$ & 0.2 & 0 & 0 & 0 \\
\hline \multicolumn{5}{|c|}{$A_{i j o p t}^{ \pm}$under 3 flow levels: } \\
\hline $\begin{array}{l}\text { Low } \\
(j=1)\end{array}$ & 0.2 & 0 & 0 & {$[3.2,4.2]$} \\
\hline $\begin{array}{l}\text { Medium } \\
(\mathrm{j}=2)\end{array}$ & 0.6 & 1 & {$[0,4]$} & 6 \\
\hline $\begin{array}{l}\text { High } \\
(j=3)\end{array}$ & 0.2 & 2.5 & 4 & 6 \\
\hline \multicolumn{3}{|c|}{ Net benefit $\left(\$ 10^{3}\right)$} & \multicolumn{2}{|c|}{$f_{\text {opt }}^{ \pm}=[360,589]$} \\
\hline
\end{tabular}

Then, the extended ITSP method is applied to the problem to retrieve the difference between the quantities of promised water-allocation target and the actual allocated water, and the obtained problems are solved. The optimal solutions of the problem are given in Table 5. Furthermore, Table 6 shows the optimal decision to choose alternatives for users under different levels of water flows.

Table 5. Optimal Solutions of the Extended ITSP Method under Optimized Water-allocation Targets $\left(10^{3} \mathrm{~m}^{3}\right)$

\begin{tabular}{|c|c|c|c|c|}
\hline & $\begin{array}{l}\text { Proba- } \\
\text { bility }\end{array}$ & $\begin{array}{l}\text { Muni } \\
\text { cipal } \\
(\mathrm{i}=1) \\
\end{array}$ & $\begin{array}{l}\text { Indus- } \\
\text { trial } \\
(\mathrm{i}=2) \\
\end{array}$ & $\begin{array}{l}\text { Agri- } \\
\text { cultural } \\
(\mathrm{i}=3)\end{array}$ \\
\hline$W_{i o p t}^{ \pm}$ & & 2.5 & 4 & 5.5 \\
\hline \multicolumn{5}{|c|}{$S_{i j \text { opt }}^{ \pm}$under 3 flow levels: } \\
\hline $\begin{array}{l}\text { Low } \\
(j=1)\end{array}$ & 0.2 & {$[2,2.5]$} & {$[3.5,4]$} & 2.3 \\
\hline $\begin{array}{l}\text { Medium } \\
(\mathrm{j}=2)\end{array}$ & 0.6 & {$[1,1.5]$} & {$[0,3.5]$} & 0 \\
\hline $\begin{array}{l}\text { High } \\
(j=3)\end{array}$ & 0.2 & 0 & 0 & 0 \\
\hline \multicolumn{5}{|c|}{$A_{\text {ijopt }}^{ \pm}$under 3 flow levels: } \\
\hline $\begin{array}{l}\text { Low } \\
(j=1)\end{array}$ & 0.2 & {$[0,0.5]$} & {$[0,0.5]$} & 3.2 \\
\hline $\begin{array}{l}\text { Medium } \\
(\mathrm{j}=2)\end{array}$ & 0.6 & {$[1,1.5]$} & {$[0.5,4]$} & 5.5 \\
\hline $\begin{array}{l}\text { High } \\
(\mathrm{j}=3)\end{array}$ & 0.2 & 2.5 & 4 & 5.5 \\
\hline \multicolumn{3}{|c|}{ Net benefit $\left(\$ 10^{3}\right)$} & \multicolumn{2}{|c|}{$f_{\text {opt }}^{ \pm}=[179,560]$} \\
\hline
\end{tabular}

Table 6. Optimal Decision to Choose Alternatives for Users under Different Levels of Water Flows

\begin{tabular}{llccc}
\hline \multicolumn{5}{c}{ Alternative $(\mathrm{k})$} \\
\hline User (i) & Flow level $(\mathrm{j})$ & $\mathrm{k}=1$ & $\mathrm{k}=2$ & $\mathrm{k}=3$ \\
\hline \multirow{3}{*}{ Municipal } & Low $(\mathrm{j}=1)$ & - & - & $\bullet$ \\
& Medium $(\mathrm{j}=2)$ & $\bullet$ & - & - \\
& High $(\mathrm{j}=3)$ & - & - & - \\
\hline \multirow{3}{*}{ Industrial } & Low $(\mathrm{j}=1)$ & $\bullet$ & - & $\bullet$ \\
& $\operatorname{Medium}(\mathrm{j}=2)$ & - & $\bullet$ & - \\
& $\operatorname{High}(\mathrm{j}=3)$ & - & - & - \\
\hline \multirow{4}{*}{ Agricultural } & $\operatorname{Low}(\mathrm{j}=1)$ & $\bullet$ & - & $\bullet$ \\
& $\operatorname{Medium}(\mathrm{j}=2)$ & - & - & - \\
& $\operatorname{High}(\mathrm{j}=3)$ & - & - & - \\
\hline
\end{tabular}

Finally, under fuzzy environments, apply Algorithm 1 to the above problem with fuzzy parameters and solve the obtained deterministic MIP problems. Its optimal solutions are reported in Tables 7 and 8.

The results of the example solved by ITSP, extended ITSP and fuzzy extended ITSP methods are depicted in Figures 2, 3, and 4. As it was shown in Figure 3, the optimized actual water 
allocation $\left(A_{i j o p t}^{ \pm}\right)$have reached the optimized water-allocation targets $\left(W_{i o p t}^{ \pm}\right)$under a seasonal high flow level. Consequently, under a high flow level, there would be no water shortage $\left(S_{13 \text { opt }}^{ \pm}=S_{23 \text { opt }}^{ \pm}=S_{33 \text { opt }}^{ \pm}=0\right.$ ) for users, water resources would be completely allocated to all users with a probability of $20 \%$, and none of retrieving alternatives would be used.

Table 7. Optimal solutions of the fuzzy extended ITSP method (in $10^{3} \mathrm{~m}^{3}$ )

\begin{tabular}{|c|c|c|c|c|}
\hline & $\begin{array}{l}\text { Proba- } \\
\text { bility }\end{array}$ & $\begin{array}{l}\text { Muni- } \\
\text { cipal } \\
(\mathrm{i}=1) \\
\end{array}$ & $\begin{array}{l}\text { Indus- } \\
\text { trial } \\
(\mathrm{i}=2)\end{array}$ & $\begin{array}{l}\text { Agri- } \\
\text { cultural } \\
(\mathrm{i}=3)\end{array}$ \\
\hline$W_{\text {iopt }}^{ \pm}$ & & 2.5 & 4 & 6 \\
\hline \multicolumn{5}{|c|}{$S_{i j \text { opt }}^{ \pm}$under 3 flow levels: } \\
\hline $\begin{array}{l}\text { Low } \\
(j=1)\end{array}$ & 0.2 & 2 & 4 & 2.28 \\
\hline $\begin{array}{l}\text { Medium } \\
(\mathrm{j}=2)\end{array}$ & 0.6 & 1.91 & 0 & 0 \\
\hline $\begin{array}{l}\text { High } \\
(j=3)\end{array}$ & 0.2 & 0 & 0 & 0 \\
\hline \multicolumn{5}{|c|}{$A_{\text {ijopt }}^{ \pm}$under 3 flow levels: } \\
\hline $\begin{array}{l}\text { Low } \\
(\mathrm{j}=1)\end{array}$ & 0.2 & 0.5 & 0 & 3.72 \\
\hline $\begin{array}{l}\text { Medium } \\
(\mathrm{j}=2)\end{array}$ & 0.6 & 0.59 & 4 & 6 \\
\hline $\begin{array}{l}\text { High } \\
(j=3)\end{array}$ & 0.2 & 2.5 & 4 & 6 \\
\hline \multicolumn{3}{|c|}{ Net benefit $\left(\$ 10^{3}\right)$} & \multicolumn{2}{|c|}{$f_{\text {opt }}^{ \pm}=[535,583]$} \\
\hline
\end{tabular}

Table 8. Optimal decision to choose alternatives under fuzzy environments

\begin{tabular}{llccc}
\hline \multicolumn{5}{c}{ Alternative $(\mathrm{k})$} \\
\hline User (i) & Flow level $(\mathrm{j})$ & $\mathrm{k}=1$ & $\mathrm{k}=2$ & $\mathrm{k}=3$ \\
\hline \multirow{3}{*}{ Municipal } & Low $(\mathrm{j}=1)$ & $\bullet$ & $\bullet$ & - \\
& Medium $(\mathrm{j}=2)$ & - & - & $\bullet$ \\
& High $(\mathrm{j}=3)$ & - & - & - \\
\hline \multirow{4}{*}{ Industrial } & Low $(\mathrm{j}=1)$ & - & $\bullet$ & $\bullet$ \\
& Medium $(\mathrm{j}=2)$ & - & - & - \\
& High $(\mathrm{j}=3)$ & - & - & - \\
\hline \multirow{4}{*}{ Agricultural } & Low $(\mathrm{j}=1)$ & $\bullet$ & - & $\bullet$ \\
& Medium $(\mathrm{j}=2)$ & - & - & - \\
& High $(\mathrm{j}=3)$ & - & - & - \\
\hline
\end{tabular}

Similarly, under a medium flow level, the total amount of water allocated to the municipal sector, the industrial unit and the agricultural sector would be $[1,1.5] \times 10^{3},[0.5,4] \times 10^{3}$, and $5.5 \times 10^{3} \mathrm{~m}^{3}$, respectively. Consequently, the solutions of $S_{12 \text { opt }}^{ \pm}=[1,1.5] \times 10^{3}, S_{22 \text { opt }}^{ \pm}=[0,3.5] \times 10^{3}$ and $S_{32 \text { opt }}^{ \pm}=0$ show that, under a medium flow level, there would be no water shortage for the agricultural sector, but the industrial unit would have to use the second alternative with available amount of $\Delta T_{22}^{ \pm}=[1,3.5] \times 10^{3} \mathrm{~m}^{3}$, and the municipal sector also would use the first alternative with available amount of $\Delta T_{11}^{ \pm}=[1,3] \times 10^{3}$ $\mathrm{m}^{3}$ for satisfying water needs.

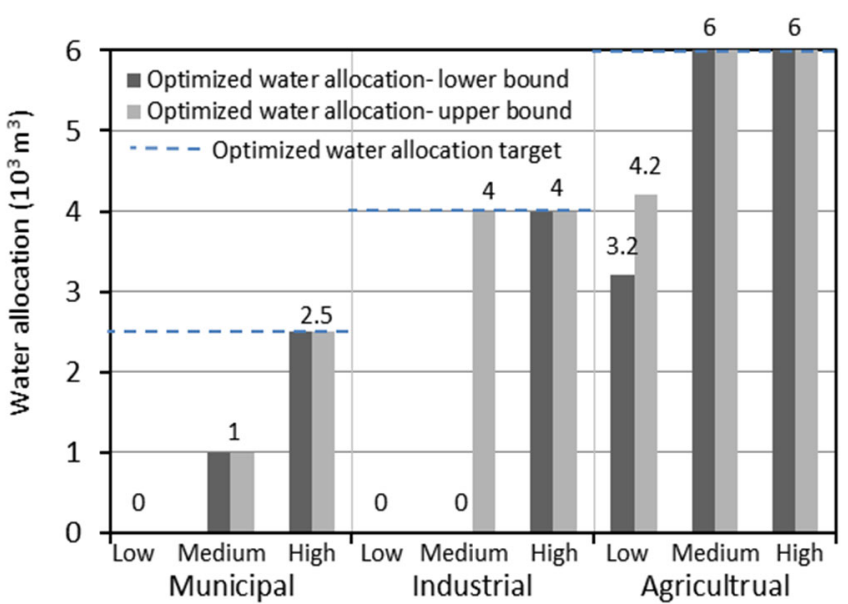

Figure 2. Optimized water-allocation patterns through the ITSP method under low, medium and high flows.

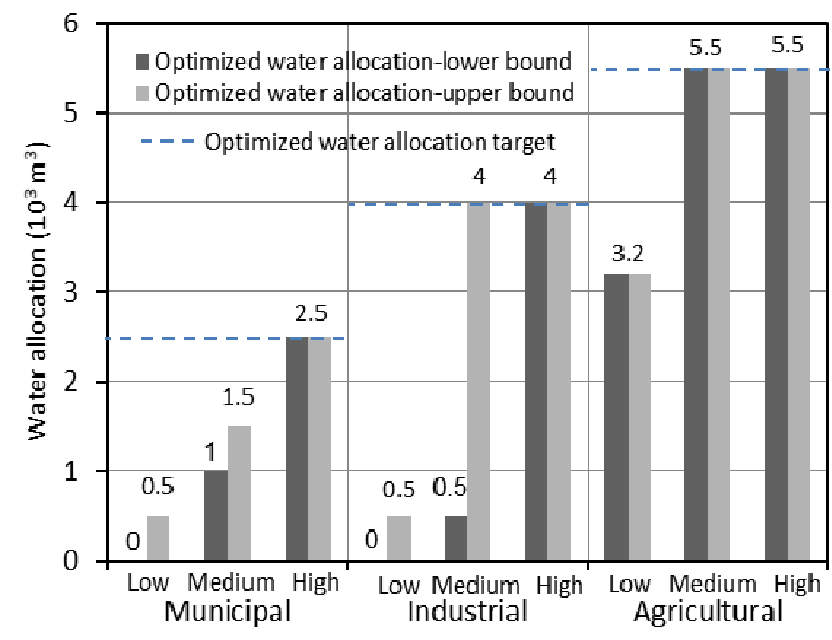

Figure 3. Optimized water-allocation patterns through the extended ITSP method under low, medium and high flows.

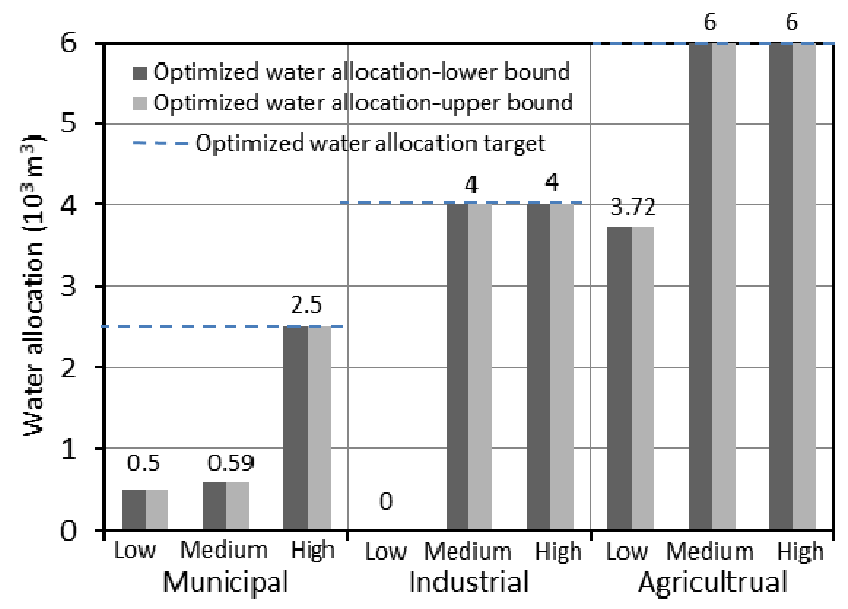

Figure 4. Optimized water-allocation patterns through the fuzzy extended ITSP method under low, medium and high flows. 
Finally, the solutions of $S_{11 \text { opt }}^{ \pm}=[2,2.5] \times 10^{3}, S_{21 \text { opt }}^{ \pm}=[3.5$, $4] \times 10^{3}$ and $S_{31 \text { opt }}^{ \pm}=2.3 \times 10^{3} \mathrm{~m}^{3}$ to water shortage under a low flow level show that all users would face water shortage and would have to use supplementary reservoirs. This happens due to insufficiencies of the water flow to satisfy optimized waterallocation target. As it was shown, a low flow level causes higher amounts of shortages that would force users to implement more than one alternative. Therefore, the best decision for the agricultural sector is to use the first and the third alternatives with available amount of $\Delta T_{31}^{ \pm}=[2,3] \times 10^{3}, \Delta T_{33}^{ \pm}=$ $[0.5,1.5] \times 10^{3} \mathrm{~m}^{3}$. The industrial unit also would use the same alternatives with available amounts of $\Delta T_{21}^{ \pm}=[0.5,2] \times 10^{3}$, $\Delta T_{23}^{ \pm}=[3,4.5] \times 10^{3} \mathrm{~m}^{3}$ and the municipal sector would only use the third alternative with amount of $\Delta T_{13}^{ \pm}=[2,5] \times 10^{3} \mathrm{~m}^{3}$ to increase water allocation amount.

By applying the fuzzy extended ITSP method to the problem, the results are shown in Figure 4 and Tables 7 and 8 . The solution of $S_{11 \text { opt }}^{ \pm}=2 \times 10^{3}, S_{12 \text { opt }}^{ \pm}=1.91 \times 10^{3}$ and $S_{13 \text { opt }}^{ \pm}=0 \mathrm{~m}^{3}$ indicate that, for the municipal sector, there would be shortages of $2 \times 10^{3}$ and $1.91 \times 10^{3} \mathrm{~m}^{3}$ under low and medium flow levels, respectively, but no water shortage under a high flow level; consequently the total amount of water allocation to the municipal sector would be $0.5 \times 10^{3}, 0.59 \times 10^{3}$, and $2.5 \times 10^{3} \mathrm{~m}^{3}$ under low, medium and high flow levels, respectively. The municipal sector would use the first and the second alternatives with available amount of $\Delta T_{11}^{ \pm}=[1,3] \times 10^{3}, \Delta T_{12}^{ \pm}=[1,3] \times 10^{3}$ $\mathrm{m}^{3}$ under a low flow level and use the third alternatives with available amount of $\Delta T_{13}^{ \pm}=[2,5] \times 10^{3} \mathrm{~m}^{3}$ under a medium flow level.

The solutions of $\left(S_{21 \text { opt }}^{ \pm}=4 \times 10^{3}, S_{22 \text { opt }}^{ \pm}=0, S_{23 \text { opt }}^{ \pm}=0 \mathrm{~m}^{3}\right)$ and $\left(S_{31 \text { opt }}^{ \pm}=2.28 \times 10^{3}, S_{32 \text { opt }}^{ \pm}=0, S_{33 \text { opt }}^{ \pm}=0 \mathrm{~m}^{3}\right)$ show that, for the industrial unit and the agricultural sector, there would be no water shortage under medium and high flow levels but there would be water shortages under a low flow level retrieved by alternatives shown in Table 8.

Under a medium flow level, only the municipal sector would face water shortage and could retrieve it by the third alternative, but two other users would not face water shortage. Furthermore, under a low flow level, all users would face water shortage and should use supplementary reservoirs.

As it was shown that, lower and upper bounds of water allocation amount are equal, so the problem gives deterministic solutions for interval variables. For different possibility levels, the results will be changed reasonably and interval values may be obtained.

Figure 5 indicates a comparison of the objective function values obtained through the general ITSP, extended ITSP and fuzzy extended ITSP methods. The system net benefit would be $\$[360,589] \times 10^{3}, \$[179,560] \times 10^{3}$ and $\$[535,583] \times 10^{3}$ for these methods respectively. The results reveal that the system net benefit decreases a little bit as using supplementary water reservoirs for water shortages. However, as it was shown that in Figure 5, the fuzzy extended ITSP method leads to the highest mid value and the smallest interval among described methods in this example.

Our fuzzy extended method has advantages in providing an effective approach to water resource management with uncertain parameters, to make appropriate decisions for retrieving water shortage, and to obtain optimal solutions with smaller intervals than the other approaches.

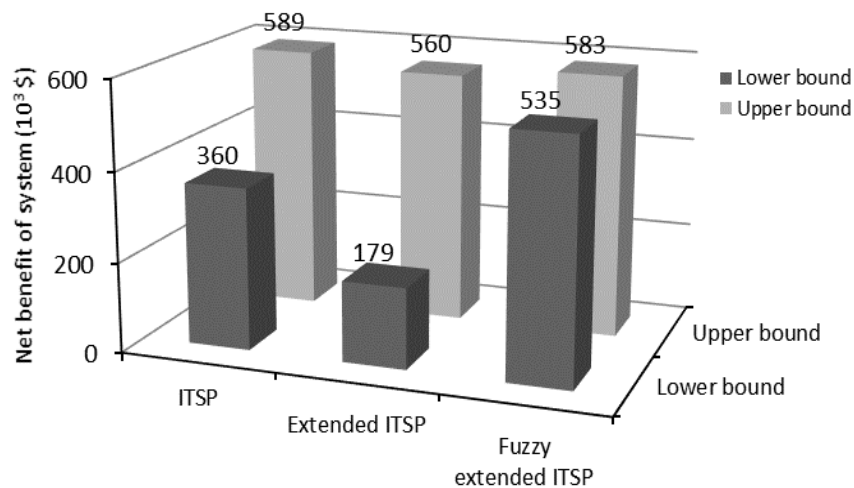

Figure 5. Comparison of upper and lower bounds of net benefits obtained through described methods.

\section{Conclusions}

In this paper, an extended ITSP method based on retrieving water shortages was introduced for water resource management. Subsequently, a fuzzy extended ITSP method has been developed for the problem under uncertainty. Then, a new method based on Huang Algorithm and FCCP method using possibility theory was proposed to solve the obtained problems.

At last by using an illustrative example, the results for the general ITSP, extended ITSP and fuzzy extended ITSP are obtained for our illustrative example to compare these results with each other and showed what the deference among these methods was.

As using supplementary water reservoirs and possibility distribution function of parameters, our fuzzy method has a higher mid value and a smaller interval than the general ITSP in the illustrative example. By considering the results, we have realized that our fuzzy extended method for water resource management leads to optimal solutions with high mid value and small intervals for objective function values and can be used to the actual cases under uncertainty. Furthermore, in our fuzzy extended ITSP method, deterministic solutions were obtained to decision variables in the illustrative example. Also, the difference between the quantities of promised water-allocation targets and the actual allocated water was retrieved by our extended ITSP approach in comparison with existing ITSP methods.

For future research, our extended approach can be developed to other uncertain variables such as random fuzzy and random rough variables and new solving methods will be obtained to the water resource management under these uncertainties. Furthermore, the other complexities that exist in water resource systems, such as nonlinear and multi-objective issues can be investigated by authors as future research.

Acknowledgments: We are grateful for anonymous referees for their constructive comments that will improve the quality of this manuscript. This research was supported by the vice-chancellor for research and technology of University of Tabriz. 


\section{Appendix}

Proof of Theorem 1.

I) Suppose that $0<\eta \leq 1$ and $\tilde{f}^{+}=\left(f^{0}, f^{1}, \beta, \gamma\right)$ then

$$
\begin{aligned}
\operatorname{Pos} & \left(\tilde{f}^{+} \geq f^{+}\right) \geq \eta \\
\Leftrightarrow \quad & \sup _{y_{1}, y_{2} \in \Re}\left\{\min \left\{\mu_{f^{+}}\left(y_{1}\right), \mu_{f^{+}}\left(y_{2}\right)\right\} \mid y_{1} \geq y_{2}\right\} \geq \eta \\
\Leftrightarrow \quad \exists y_{1}, y_{2}\left(y_{1} \geq y_{2}\right): & \\
& L\left(\frac{f^{0}-y_{1}}{\beta}\right) \geq \eta, R\left(\frac{y_{1}-f^{1}}{\gamma}\right) \geq \eta, y_{2}=f^{+} \\
\Leftrightarrow \quad & \exists y_{1}, y_{2}\left(y_{1} \geq y_{2}\right): \\
& f^{0}-L^{*}(\eta) \beta \leq y_{1} \leq f^{1}+R^{*}(\eta) \gamma, y_{2}=f^{+} \\
\Leftrightarrow \quad & \sum_{i=1}^{m}\left(N B_{i}^{1}+R^{*}(\eta) \lambda_{i}\right)\left(W_{i}^{-}+\Delta W_{i} z_{i}\right) \\
& +\sum_{i=1}^{m} \sum_{j=1}^{n} p_{j}\left(-C_{i}^{0}+R^{*}(\eta) \rho_{i}\right) S_{i j}^{-} \\
& +\sum_{i=1}^{m} \sum_{j=1}^{n} p_{j} \sum_{k=1}^{l}\left(-E_{i k}^{0}+R^{*}(\eta) \alpha_{i k}\right) \Delta T_{i k}^{-} x_{i j k} \geq f^{+} .
\end{aligned}
$$

II) Let $0<h \leq 1$ and $\tilde{a}_{j}^{-}=\sum_{i=1}^{m}\left(W_{i}^{-}+\Delta W_{i} z_{i}-S_{i j}^{-}\right)(1+\tilde{\delta})$

then

$$
\begin{aligned}
& \operatorname{Pos}\left(\sum_{i=1}^{m}\left(W_{i}^{-}+\Delta W_{i} z_{i}-S_{i j}^{-}\right)(1+\tilde{\delta}) \leq \tilde{q}_{j}\right) \geq h \\
& \Leftrightarrow \sup _{y_{1}, y_{2} \in \Re}\left\{\min \left\{\mu_{\tilde{a}_{j}^{-}}\left(y_{1}\right), \mu_{\tilde{q}_{j}}\left(y_{2}\right)\right\} \mid y_{1} \leq y_{2}\right\} \geq h
\end{aligned}
$$

\section{References}

Birge, J.R., and Louveaux, F.V. (1988). A multicut algorithm for twostage stochastic linear programs. Eur. J. Oper. Res., 34(3), 384-392. http://dx.doi.org/10.1016/0377-2217(88)90159-2

Eiger, G., and Shamir, U. (1991). Optimal operation of reservoirs by stochastic programming. Eng. Optimiz., 17(4), 293-312. http:// dx.doi.org/10.1080/03052159108941077

Fan, Y.R., Huang, G.H., Guo, P., and Yang, A.L. (2012). Inexact twostage stochastic partial programming: Application to water resources management under uncertainty. Stochastic Environ. Res. Risk Assess., 26(2), 281-293. http://dx.doi.org/10.1007/s00477-0110504-6

Ferrero, R.W., Rivera, J.F., and Shahidehpour, S.M. (1998). A dynamic programming two stage algorithm for long-term hydrothermal scheduling of multireservoir systems. IEEE Trans. Power Syst., 13(4), 1534-1540. http://dx.doi.org/10.1109/59.736302

Guo, P., Huang, G.H., He, L., and Sun, B.W. (2008). ITSSIP: Intervalparameter two-stage stochastic semi-infinite programming for environmental management under uncertainty. Environ. Model. Software, 23(12), 1422-1437. http://dx.doi.org/10.1016/j.envsoft.2008. 04.009

Guo, P., Huang, G.H., Zhu, H., and Wang, X.L. (2010). A two-stage programming approach for water resources management under randomness and fuzziness. Environ. Model. Software, 25(12), 15731581. http://dx.doi.org/10.1016/j.envsoft.2010.04.018

$$
\begin{aligned}
\Leftrightarrow & \exists y_{1}, y_{2}\left(y_{1} \leq y_{2}\right): \\
& L\left(\frac{\sum_{i=1}^{m}\left(W_{i}^{-}+\Delta W_{i} z_{i}-S_{i j}^{-}\right)\left(1+\delta^{0}\right)-y_{1}}{\sum_{i=1}^{m}\left(W_{i}^{-}+\Delta W_{i} z_{i}-S_{i j}^{-}\right) \beta}\right) \geq h, \\
& R\left(\frac{y_{1}-\sum_{i=1}^{m}\left(W_{i}^{-}+\Delta W_{i} z_{i}-S_{i j}^{-}\right)\left(1+\delta^{1}\right)}{\sum_{i=1}^{m}\left(W_{i}^{-}+\Delta W_{i} z_{i}-S_{i j}^{-}\right) \gamma}\right) \geq h, \\
& L\left(\frac{q_{j}^{0}-y_{2}}{\beta_{j}}\right) \geq h, R\left(\frac{y_{2}-q_{j}^{1}}{\gamma_{j}}\right) \geq h \\
\Leftrightarrow & \exists y_{1}, y_{2}\left(y_{1} \leq y_{2}\right): \\
& \sum_{i=1}^{m}\left(W_{i}^{-}+\Delta W_{i} z_{i}-S_{i j}^{-}\right)\left(1+\delta^{0}\right) \\
& -L^{*}(h) \sum_{i=1}^{m}\left(W_{i}^{-}+\Delta W_{i} z_{i}-S_{i j}^{-}\right) \beta \leq y_{1} \\
& \leq \sum_{i=1}^{m}\left(W_{i}^{-}+\Delta W_{i} z_{i}-S_{i j}^{-}\right)\left(1+\delta^{1}\right) \\
& +R^{*}(h) \sum_{i=1}^{m}\left(W_{i}^{-}+\Delta W_{i} z_{i}-S_{i j}^{-}\right) \gamma, \\
& q_{j}^{0}-L^{*}(h) \beta_{j} \leq y_{2} \leq q_{j}^{1}+R^{*}(h) \gamma_{j} \\
\Leftrightarrow & \sum_{i=1}^{m}\left(W_{i}^{-}+\Delta W_{i} z_{i}-S_{i j}^{-}\right)\left(1+\delta^{0}-L^{*}(h) \beta\right. \\
& \leq q_{j}^{1}+R^{*}(h) \gamma_{j} .
\end{aligned}
$$

Hu, Q., Huang, G., Liu, Z., Fan, Y., and Li, W. (2012). Inexact fuzzy two-stage programming for water resources management in an environment of fuzziness and randomness. Stochastic Environ. Res. Risk Assess., 26(2), 261-280. http://dx.doi.org/10.1007/s00477-0110503-7

Huang, G.H. (1996). IPWM: An interval parameter water quality management model. Eng. Optimiz., 26(2), 79-103. http://dx.doi.org/ $10.1080 / 03052159608941111$

Huang, G.H., and Loucks, D.P. (2000). An inexact two-stage stochastic programming model for water resources management under uncertainty. Civ. Eng. Environ. Syst., 17(2), 95-118. http://dx.doi.org/ 10.1080/02630250008970277

Huang, G.H., and Chang, N.B. (2003). The perspectives of environmental informatics and systems analysis. J. Environ. Inf., 1(1), 1-7. http://dx.doi.org/10.3808/jei.200300001

Jairaj, P.G., and Vedula, S. (2000). Multireservoir system optimization using fuzzy mathematical programming. Water Resour. Manage., 14(6), 457-472. http://dx.doi.org/10.1023/A:1011117918943

Jiménez, M., Arenas, M., Bilbao, A., and Rodríguez, M.W. (2007). Linear programming with fuzzy parameters: An interactive method resolution. Eur. J. Oper. Res., 177(3), 1599-1609. http://dx.doi.org/ 10.1016/j.ejor.2005.10.002

Li, Y.P., Huang, G.H., and Nie, S.L. (2006). An interval-parameter multi-stage stochastic programming model for water resources management under uncertainty. Adv. Water Resour., 29(5), 776-789. http://dx.doi.org/10.1016/j.advwatres.2005.07.008 
Li, Y.P., Huang, G.H., Nie, S.L., and Qin, X.S. (2007). ITCLP: An inexact two-stage chance-constrained program for planning waste management systems. Resour. Conserv. Recycling, 49(3), 284-307. http://dx.doi.org/10.1016/j.resconrec.2006.03.017

Li, Y.P., and Huang, G.H. (2008). Interval-parameter two-stage stochastic nonlinear programming for water resources management under uncertainty. Water Resour. Manage., 22(6), 681-698. http:// dx.doi.org/10.1007/s11269-007-9186-8

Maqsood, I., Huang, G.H., and Scott Yeomans, J. (2005). An intervalparameter fuzzy two-stage stochastic program for water resources management under uncertainty. Eur. J. Oper. Res., 167(1), 208-225. http://dx.doi.org/10.1016/j.ejor.2003.08.068

Nie, X.H., Huang, G.H., Li, Y.P., and Liu, L. (2007). IFRP: A hybrid interval-parameter fuzzy robust programming approach for waste management planning under uncertainty. J. Environ. Manage. 84(1), 1-11. http://dx.doi.org/10.1016/j.jenvman.2006.04.006

Ruszczyński, A., and Swiettanowski, A. (1997). Accelerating the regularized decomposition method for two-stage stochastic linear problems. Eur. J. Oper. Res., 101(2), 328-342. http://dx.doi.org/10.1016/ S0377-2217(96)00401-8

Wang, D., and Adams, B.J. (1986). Optimization of real-time reservoir operations with Markov decision processes. Water Resour. Res. 22(3), 345-352. http://dx.doi.org/10.1029/WR022i003p00345
Wang, L.Z., Fang, L., and Hipel, K.W. (2003). Water resources allocation: A cooperative game theoretic approach. J. Environ. Inf., 2(2), 11-22. http://dx.doi.org/10.3808/jei.200300019

Wang, S., Huang, G.H., Lu, H.W., and Li, Y.P. (2011). An intervalvalued fuzzy linear programming with infinite $\alpha$-cuts method for environmental management under uncertainty. Stochastic Environ. Res. Risk Assess., 25(2), 211-222. http://dx.doi.org/10.1007/s00477 -010-0432-x

Wang, S., and Huang, G.H. (2011). Interactive two-stage stochastic fuzzy programming for water resources management. J. Environ. Manage., 92(8), 1986-1995. http://dx.doi.org/10.1016/j.jenvman. 2011.03.024

Wang, S., and Huang, G.H. (2015). A multi-level Taguchi-factorial two-stage stochastic programming approach for characterization of parameter uncertainties and their interactions: An application to water resources management. Eur. J. Oper. Res., 240(2), 572-581. http://dx.doi.org/10.1016/j.ejor.2014.07.011

Wang, S., and Watada, J. (2011). Two-stage fuzzy stochastic programming with Value-at-Risk criteria. Appl. Soft Comput., 11(1), 10441056. http://dx.doi.org/10.1016/j.asoc.2010.02.004 